Rev. IG, São Paulo, 1(1):53-68, jan./jun. 1980

\title{
UM MÉTODO PARA AVALIAÇÃO APROXIMADA DE COEFICIENTES DE PERMEABILIDADE EM AQÜIFFEROS
}

\author{
Eng. ${ }^{\circ}$ MOACYR DE CARVALHO
}

\begin{abstract}
The present work is an attempt to obtain approximate values of aquifer permeability coefficients whose structure often exhibit bedding.

The method consist in its essence, through some restrictive conditions, in the establishment of a linear equation system.

For this purpose it is utilized known data of tubular wells within a limited area, under the hypothesis that each aquifer strata behave as an individual free water table.

An example the applicability, it is presented using data draw out of three tubular wells from the town of Catanduva.

Nevertheless, the wells have an imaginary sedimentary strata due to a lack of an adequated geological profiles description.

They does not follow the restrictive conditions established on such method, because the water contributions from underlying fractured rocks, should have come probably, from Botucatu Formation.
\end{abstract}

\section{RESUMO}

O presente trabalho é uma tentativa para a obtenção de valores aproximados de coeficiente de permeabilidade de aqǘf́eros em cuja estrutura ocorrem camadas ou estratos diversos de sedimentos.

O método consiste na essência, partindo de certas condições restritivas, no estabelecimento de um sistema de equações lineares utilizando-se de dados conhecidos de poços tubulares numa área limitada, sob a hipótese de que cada estrato do aqüífero se comporte como um aqüífero individual de lençol livre.

Um exemplo de aplicação é apresentado com dados retirados de 3 poços tubulares da cidade de Catanduva, porém com estratificações sedimentares imaginárias devido a inexistência de uma descrição adequada dos perfís geológicos. Observamos também que tais poços não obedecem as condições restritivas impostas no método, por receberem contribuições de rochas fraturadas subjacentes, provavelmente de água proveniente da Formação Botucatu.

\section{INTRODUÇÃO}

Atualmente a busca de água ocorrente em lençóis subterrâneos tem crescido em todo o mundo em função da expansão demográfica, da atividade econômica e ainda como conseqüência da poluição dos cursos d'água de superfície.
Paralelamente à expansão da demanda no uso da água para fins múltiplos, vem sendo imprimido um ritmo mais acelerado nas questões que dizem respeito à pesquisa de água subterrânea, envolvendo no contexto uma política em torno da racionalização do seu uso.

Estes aspectos têm condicionado a necessidade de investigações cada vez mais 
precisas no dimensionamento das reservas de água de origem subterrânea e no conhecimento mais detalhado dos aqǘf́eros, para o estabelecimento do modelo que o representa em estrutura e da dinâmica da água que por ela circula, como um segmento do ciclo hidrológico que é.

No Estado de São Paulo ocorrem duas formações geológicas extremamente importantes que, por suas características, vêm sendo procuradas como fonte de abastecimento de água, como um meio de solução para graves problemas que afetam principalmente populações de centros urbanos. São elas a Formação Bauru e a Formação Botucatu. Contam-se já por milhares os poços tubulares perfurados, notadamente na primeira estrutura sedimentar, sem o necessário cadastramento e controle, principais instrumentos de uma política de racionalização do uso de água de origem subterrânea.

\section{OBJETIVO}

Este trabalho tem por finalidade tentar desenvolver uma metodologia orientada no sentido de obter, por via indireta, uma avaliação do débito de um poço tubular, mediante elementos de outros poços já perfurados numa área restrita, nas condições que abaixo serão impostas.

Hoje o método para a avaliação de vazões de poços tubulares parte de dados conhecidos de outros poços relativamente próximos e do conhecimento da estrutura geológica da região, conhecimento este sempre baseado em extrapolações. Assim o perfil geológico e a vazão reais som?nte são conhecidos após a perfuração e o bombeamento de teste de produção.

A proximidade relativa de poços tubulares é freqüente, principalmente nos centros urbanos que se servem de água subterrânea para fins de abastecimento público e industrial, fato este comum nas cidades implantadas na Formação Bauru.
Mais precisamente, o que se quer é a medida de valores de permeabilidade por modelo matemático das várias camadas dè sedimento de aqǘf́eros a partir de um certo número de poços tubulares que, por condições de proximidade e de comportamento hidráulico faça supor um certo grau de homogeneidade estrutural, evidentemente válidas dentro de limites restritos.

\section{METODOLOGIA}

A metodologia aqui aplicada, na dedução de coeficientes de permeabilidade, consiste na obtenção de um sistema linear de equações de $\mathbf{n}$ incógnitas $\left(\mathrm{k}_{1}, \mathrm{k}_{2} \ldots \mathrm{K}_{\mathrm{n}}\right)$, a partir de $\mathbf{n}$ poços perfurados com vazões de teste conhecidos: $\mathrm{Q}_{1}, \mathrm{Q}_{2}, \ldots \ldots \mathrm{Q}_{\mathrm{n}}$, com camadas de sedimentos reconhecidos e de espessuras $H_{1}-h_{1}$ determinadas, que podem ser conseguidas no curso da própria perfuração.

O poço será considerado como tendo raio constante e um raio de influência $R$ que será retirado de tabelas a partir do conhecimento da fração granulométrica principal do sedimento.

Nestas condições, tendo-se n camadas de sedimentos, ter-se-ão os correspondentes coeficientes $\mathrm{K}_{1}, \mathrm{~K}_{2} \ldots \ldots \mathrm{K}_{\mathrm{n}}$ de permeabilidade, não todos nulos. Supõe-se que os coeficientes $k_{i}$ de camadas argilosas são todos nulos, devido a baixa permeabilidade das argilas.

Admite-se também que em cada camada identificada do aquíf́fero é válida a expressão de Dupuit:

$$
\mathrm{Q}=2 \pi \mathrm{xyk} \frac{\mathrm{dy}}{\mathrm{dx}}
$$

deduzida para um poço perfeito para o cálculo do afluxo de água em um lençol livre.

Consideramos que a vazão de teste pode ser expressa pela fórmula:

$$
\begin{gathered}
\mathrm{Q}=\frac{\pi \mathrm{k}\left(\mathrm{H}^{2}-\mathrm{h}^{2}\right)}{1 \mathrm{nR}-1 \mathrm{nr}}=\frac{\pi \mathrm{k} \mathrm{dd}^{\prime}}{1 \mathrm{nR}-1 \mathrm{nr}}=\mathrm{ak} \\
\mathrm{a}=\frac{\pi \mathrm{Dd}}{1 \mathrm{nR}-1 \mathrm{nr}}
\end{gathered}
$$


onde:

$\mathrm{D}=\mathrm{H}-\mathrm{h}$ espessura da camada de sedimento a contar do plano horizontal que passa pelo fundo do poço que a limita inferiormente

$\mathrm{d}=\mathrm{H}+\mathrm{h}$

$\mathrm{R}$ raio de influência

Para as camadas de sedimentos distintos, compondo o aqüífero, a vazão de teste poderá ser decomposta de acordo com a expressão:

$\mathrm{Q}=\Delta \mathrm{Q}_{1}+\Delta \mathrm{Q}_{2}+\ldots \ldots+\Delta \mathrm{Q}_{\mathrm{n}}$

onde os $\mathrm{Q}_{\mathrm{i}}$ representam a contribuição isolada de cada camada de sedimentos.

Imaginemos a superfície de um aqüífero de dimensões reduzidas, com certo grau de homogeneidade, onde se encontram n poços tubulares com vazões de teste conhecidas:

$$
\mathrm{Q}_{1}, \mathrm{Q}_{2}, \ldots \ldots \mathrm{Q}_{\mathrm{n}} \text {. }
$$

Pelas hipóteses admitidas pode-se ter n incógnitas, de solução única, isto é, admite apenas o sistema único $\mathrm{k}_{1}, \mathrm{k}_{2} \ldots \mathrm{k}_{\mathrm{n}}$. Se

$\mathrm{Q}=\Sigma \mathrm{Q}_{\mathrm{i}}$ e $\mathrm{Q}_{\mathrm{i}}=\mathrm{a}_{\mathrm{ik}} \mathrm{K}_{\mathrm{i}} \mathrm{i}, \mathrm{k}=(1,2, \ldots$ (....n)

então tem-se o sistema linear:

$\mathrm{a}_{11} \mathrm{k}_{1}+\mathrm{a}_{12} \mathrm{k}_{2}+\ldots \ldots+\mathrm{a}_{1 \mathrm{n}} \mathrm{k}_{\mathrm{n}}=\mathrm{Q}_{1}$ $\mathrm{a}_{21} \mathrm{k}_{1}+\mathrm{a}_{22} \mathrm{k}_{2}+\ldots \ldots+\mathrm{a}_{2 \mathrm{n}} \mathrm{k}_{\mathrm{n}}=\mathrm{Q}_{2}$

$\mathrm{a}_{\mathrm{n} 1} \mathrm{~K}_{1}+\mathrm{a}_{\mathrm{n} 2} \mathrm{k}_{2}+\ldots \ldots+\mathrm{a}_{\mathrm{nn}} \mathrm{k}_{\mathrm{n}}=\mathrm{Q}_{\mathrm{n}}$

Usando-se a notação da álgebra matricial vem:

$$
\text { A } \mathrm{K}=\mathrm{Q}
$$

onde

$$
\begin{aligned}
& A=\left[\begin{array}{lllll}
a_{11} & a_{12} & \ldots & \ldots & a_{1 n} \\
a_{21} & a_{22} & \ldots & \ldots & a_{2 n} \\
a_{31} & a_{32} & \ldots & \ldots & a_{3 n} \\
\ldots & \ldots & \ldots & \ldots & \\
a_{n 1} & a_{n 2} & \ldots & \ldots & a_{n n}
\end{array}\right] \\
& \mathrm{K}=\left[\begin{array}{c}
\mathrm{k}_{1} \\
\mathrm{k}_{2} \\
\mathrm{k}_{3} \\
\vdots \\
\mathrm{k}_{\mathrm{n}}
\end{array}\right] \quad \mathrm{Q}=\left[\begin{array}{c}
\mathrm{Q}_{1} \\
\mathrm{Q}_{2} \\
\mathrm{Q}_{3} \\
\vdots \\
\mathrm{Q}_{\mathrm{n}}
\end{array}\right]
\end{aligned}
$$

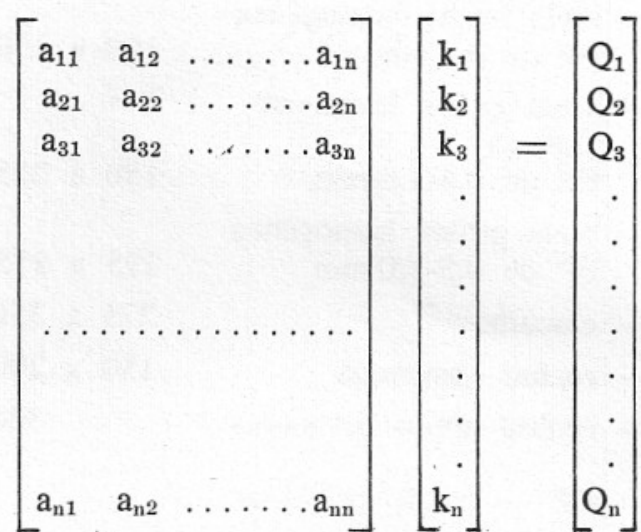

Os elementos da matriz quadrada A são expressos por

$$
\begin{aligned}
& \mathrm{a}_{\mathrm{ik}}=\frac{\left(\mathrm{H}_{\mathrm{i}}-\mathrm{h}_{\mathrm{i}}\right)\left(\mathrm{H}_{\mathrm{i}}+\mathrm{h}_{\mathrm{i}}\right) \pi}{\ln \mathrm{R}-\mathrm{ln} \mathrm{r}} \text { e assim } \\
& \mathrm{Q}_{\mathrm{i}}=\mathrm{a}_{\mathrm{ik}} \mathrm{k}_{\mathrm{i}} \quad \begin{array}{l}
\text { vazão de cada camada do } \\
\text { aquífero }
\end{array} \\
& \mathrm{H}_{\mathrm{i}} \text { e } \mathrm{h}_{\mathrm{i}} \begin{array}{l}
\text { é a altura dos planos que limi- } \\
\text { tam superior e inferiormente } \\
\text { cada camada } i \text { de sedimentos }
\end{array}
\end{aligned}
$$

As alturas $\mathrm{H}_{\mathrm{i}}$ e $\mathrm{h}_{1}$ sempre serão referidas ao plano que passa pelo fundo do poço e limita inferiormente o aqüífero.

Assinalamos desde já que os valores dos coeficientes de permeabilidade obtidos por bombeamento diferem dos valores obtidos por injeção de água, tendendo os primeiros a apresentarem valores mais baixos, o que é compreensível.

\section{TABELA DE R (m), RAIO}

DE INFLUENCIA

\section{TERRENO}

- vaza arenosa

- areia fina argilosa FP de 0,01-0,05 mm

- areia fina homogênea FP de 0,01-0,05 mm

- areia fina argilosa FP de $0,1-0,25 \mathrm{~mm}$

- areia fina homogênea FP de 0,1-0,25 mm

- areia média argilosa FP de 0,25-0,5 mm

\section{$\mathrm{R}(\mathrm{m})$}

10 a 20

30 a 40

40 a 50

50 a 60

60 a 80

80 a 120 
- areia média homogênea FP de $0,5 \mathrm{~mm}$

100 a 150

- areia grossa levemente argilosa

FP de 0,5-1,0 mm

150 a 225

- areia grossa homogênea FP de $0,5-1,0 \mathrm{~mm}$

225 a 275

275 a 350

- cascalho

150 a 200

- rochas fissuradas 500

FP - fração principal

OBS.: Esta tabela foi extraída da obra "Hydrogéologie et notions de geologie d'ingénieur"

\section{CONCLUSÃO}

Iniciemos esta conclusão com uma ligeira discussão da metodologia proposta.

Designemos por d (A) o determinante associado à matriz quadrada $\mathrm{A}$. Para que o sistema (1) tenha solução é necessário e suficiente que $d(A) \neq 0$.

\section{Então:}

$\mathrm{Ak}=\mathrm{Q}$ e $\mathrm{k}=\mathrm{A}^{-1} \mathrm{Q}$ onde a determinação do inverso da matriz $\mathrm{A}, \mathrm{A}^{-1}$ exige a condição $d(A) \neq 0$.

Os elementos $\mathrm{a}_{\mathrm{ik}}$ da matriz $\mathrm{A}$ são todos positivos por razões geométricas e físicas, isto é, $a_{i k} \geqslant 0$.

Também os coeficientes de permeabilidade $k_{i}$ são maiores ou iguais a zero. Logo todos os termos da esquerda das equações do sistema são positivos. Assim se no sistema (1) ocorrer que dois ou mais valores $Q_{i}$ sejam idênticos, as equações correspondentes serão idênticas e o sistema se reduz a um sistema de menor número de equações.

Também $\mathrm{k}_{\mathrm{i}}=0$ (caso da permeabilidade das argilas) o número de equações se reduz igualmente pela redução do número de incognitas $\mathrm{k}_{\mathrm{i}}$. Assim, para que o sistema (1) seja compatível e determinada, de solução única, as duas condições abaixo deverão estar satisfeitas

$\mathrm{d}(\mathrm{A}) \neq 0$ e $\mathrm{Q}_{\mathrm{i}} \neq \mathrm{Q}_{2} \neq \mathrm{Q}_{3} \neq \ldots \neq \mathrm{Q}_{\mathrm{n}}$

A não satisfação destas condições é apenas uma possibilidade teórica.
O trabalho aqui desenvolvido é, na realidade, uma tentativa de generalização da equação de Dupuit para a determiniação de $\mathrm{k}$ a partir de dois poços testemunhos:

$$
\begin{aligned}
& \mathrm{k}=\frac{\mathrm{Q}\left({ }^{1} \mathrm{na}_{2}-1 \mathrm{na}_{1}\right)}{2 \mathrm{M}\left(\mathrm{h}_{2}-\mathrm{h}_{1}\right)} \\
& \mathrm{M}=\text { espessura do aquífero }
\end{aligned}
$$

sendo $\mathrm{Q}, \mathrm{h}_{1}$ e $\mathrm{h}_{2}$ a água bombeada e os níveis nos dois poços testemunhos distantes de $a_{1}$ e $a_{2}$ do poço central ensaiado. Assim, pode-se calcular o coeficiente $\mathrm{k}$ em um lençol aqüífero livre.

Entretanto se um poço tubular atravessa um aqüífero que recebe contribuição de várias camadas de sedimentos com valores $\mathrm{Q}_{\mathrm{i}}$, a determinação de $\mathrm{k}$ pela fórmula indicada daria um valor médio do coeficiente de permeabilidade, já que neste caso se exprimira pela soma $\mathrm{k}=\mathrm{k}_{1}+\mathrm{k}_{2}$ $+\ldots \ldots \ldots \mathrm{k}_{\mathrm{n}}$. Isto foi admitido na formulação do sistema (1).

Quanto ao raio de influência $\mathrm{R}$ que integra a expressão dos elementos $a_{i \mathbf{k}}$ da matriz A, as fórmulas existentes para a sua determinação são imprecisas, porém aceitáveis.

Aliás deve-se ter em conta que as leis que descrevem os movimentos da água, em Hidráulica, são freqüentemente afetadas de coeficientes corretivos obtidos experimentalmente. Desta maneira qualquer desenvolvimento teórico do fluxo de fluidos, objeto da Hidrodinâmica, ao passar para as aplicações práticas tem necessidade de ser testado, tarefa essa nem sempre fácil ou possível.

A comprovação do método deduzido dependerá, pois, de um teste, embora se fundamente em equações correntes na prática da hidrogeologia.

É ainda de se esperar que, em razão da complexidade do arranjo, dimensões e forma das partículas de sedimentos que compõem as formações geológicas sedimentares, os coeficientes ki de permeabilidade calculados sejam de uma natureza probabilística ou freqüencista. Em vista disso, a abordagem correta do problema deveria se realizar através da teoria Matemática da Probabilidade. Por exemplo, a clássica formula de Darcy para a avaliação de perdas de carga ou de energia específica está hoje superada em precisão pela fórmula de 
Hazen-Willians obtida estatisticamente, para não citar outros exemplos da Física Estatística.

Em que pesem tais considerações, é provável que se possa obter precisão razoável na avaliação prévia de produção de poços a perfurar, através do método aqui apresentado. Também, dada a simplicidade, a estrutura ou modelo de aqüíferos poderá ser construído por esta via.

Nota-se que, se no aqǘfero atravessado por poços tubulares ocorrem camadas de argila, desde logo ficam alijadas do sistema várias incógnitas e, portanto, este se simplifica.

Sendo a matriz quadrada $\mathrm{A}$ composta de pequeno número de elementos, via de regra, o sistema (1) pode ser resolvido em uma pequena calculadora eletrônica programável como a Hewlett Packard modelo 9820 A, opção 001.

Dada a existência de perspectiva de aplicação de uma tal metodologia, seria de conveniência não somente para propósito como para outras investigaçõ̃es futuras, que os poços tubulares perfurados por este Instituto Geológico bem como por outras entidades, fosse providenciada a locação dos mesmos através de coordenadas do sistema UTM, o que pode ser conseguido ao nível de precisão permissível, usando-se folhas topográficas na escala de 1:50.000, hoje disponíveis para todo o Estado de São Paulo.
A descrição e a espessura dos sedimentos atravessados são também dados de extrema importância no aprofundamento de pesquisas hidrogeológicas, bem como a determinação da granulometria de amostras representativas das camadas de sedimentos, dados esses que contribuem para a construção do modelo de aqüíferos.

A aplicação sucessiva do método apresentado concorrerá para que os resultados encontrados se aproximem por iterações aos do modelo real.

Outro aspecto positivo que poderá decorrer da aplicação de modelos matemáticos é o desenvolvimento de métodos indiretos, cada vez mais necessários às investigações hidrogeológicas, tendo em consideração os custos e o tempo despendido pela via direta.

Esta é uma abordagem que vem crescendo rapidamente como consequiência do desenvolvimento de vários ramos da matemática aplicada e principalmente dos computadores eletrônicos.

Para a compatibilização das unidades que entram nos termos das equações do sistema (1), observando-se que os valores de permeabilidade são expressos habitualmente em metro/dia, é necessário que as outras grandezas sejam expressas em metro $\mathrm{e}$, os valores de vazão $\mathrm{Q}$, ao invés de $\mathrm{m}^{3}$ / hora como é corrente em testes de vazão, se expressem $\mathrm{em} \mathrm{m}^{3} /$ dia.

\section{DADOS PARA OS CÁLCULOS DOS COEFICIENTES $\alpha_{i \mathbf{k}}$}

POÇO 1

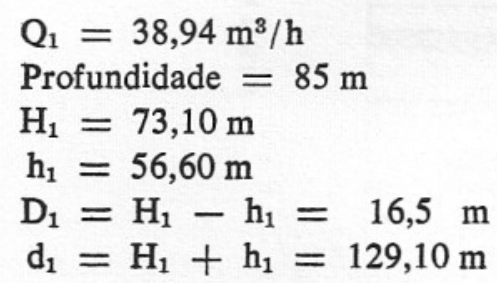

POÇO 2

$$
\begin{aligned}
& \mathrm{Q}_{2}=22,23 \mathrm{~m}^{3} / \mathrm{h} \\
& \text { Profundidade }=85 \mathrm{~m} \\
& \mathrm{H}_{2}=67 \mathrm{~m} \\
& \mathrm{~h}_{2}=57 \mathrm{~m} \\
& \mathrm{D}_{2}=\mathrm{H}_{2}-\mathrm{h}_{2}=10 \mathrm{~m} \\
& \mathrm{~d}_{2}=\mathrm{H}_{2}+\mathrm{h}_{2}=124 \mathrm{~m}
\end{aligned}
$$

$\begin{array}{ll}1_{11}=21 \mathrm{~m} & \mathrm{R}_{1}=40 \mathrm{~m} \\ 1_{12}=25 \mathrm{~m} & \mathrm{R}_{2}=42 \mathrm{~m} \\ 1_{13}=27,10 \mathrm{~m} & \mathrm{R}_{3}=45 \mathrm{~m}\end{array}$

$1_{21}=20 \mathrm{~m}$

$\mathrm{R}_{1}=40 \mathrm{~m}$

$1_{22}=23 \mathrm{~m}$

$\mathrm{R}_{2}=42 \mathrm{~m}$

$1_{23}=24 \mathrm{~m}$

$\mathrm{R}_{3}=45 \mathrm{~m}$ 
Rev. IG, São Paulo, 1(1):53-68, jan./jun. 1980

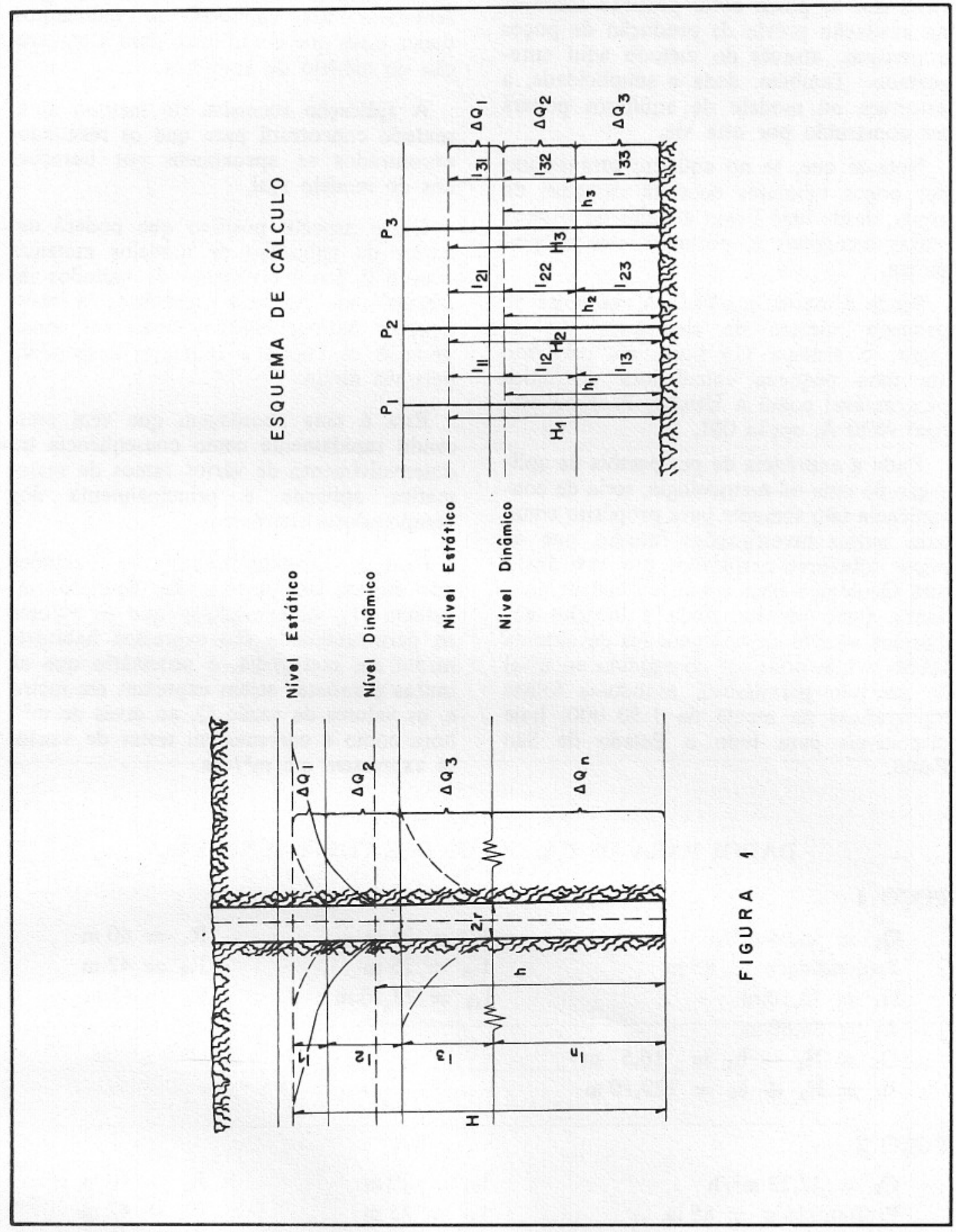


Rev. IG, Săo Paulo, 1(1):53-68, jan./jun. 1980

\section{POÇO 3}

$$
\begin{aligned}
\mathrm{Q}_{3} & =18,37 \mathrm{~m}^{3} / \mathrm{h} & 1_{31}=20,7 \mathrm{~m} & \mathrm{R}_{1}=40 \mathrm{~m} \\
\mathrm{H}_{3} & =62,10 \mathrm{~m} & 1_{32}=22,7 \mathrm{~m} & \mathrm{R}_{2}=42 \mathrm{~m} \\
\mathrm{~h}_{3} & =52,40 \mathrm{~m} & 1_{33}=18,7 \mathrm{~m} & \mathrm{R}_{3}=45 \mathrm{~m} \\
\mathrm{D}_{3} & =\mathrm{H}_{3}-\mathrm{h}_{3}=9,70 \mathrm{~m} & & \\
\mathrm{~d}_{3} & =\mathrm{H}_{3}+\mathrm{h}_{3}=114,5 \mathrm{~m} & &
\end{aligned}
$$

\section{CÁLCULO DOS COEFICIENTES $\alpha_{\mathrm{ik}}$}

$$
a_{11}=\frac{3,14 \times 16,5 \times 129,1}{24(73,10)^{2}}
$$

$$
a_{12}=\frac{3,14 \times 16,5 \times 129,1}{24(73,10)^{2}}
$$

$$
a_{13}=\frac{3,14 \times 16,5 \times 129,1}{24(73,10)^{2}}
$$

$$
\mathrm{a}_{21}=\frac{3,14 \times 10 \times 124}{24(67)^{2}}
$$$$
a_{22}=\frac{3,14 \times 10 \times 124}{24(67)^{2}}
$$$$
a_{23}=\frac{3,14 \times 10 \times 124}{24(67)^{2}}
$$$$
a_{31}=\frac{3,14 \times 9,7 \times 114,5}{24(62,10)^{2}}
$$$$
a_{32}=\frac{3,14 \times 9,7 \times 114,5}{24(62,10)^{2}}
$$$$
a_{33}=\frac{3,14 \times 9,7 \times 114,5}{24(62,10)^{2}}
$$

$$
\frac{21^{2}}{\ln 40-\ln 0,10}=3,85
$$
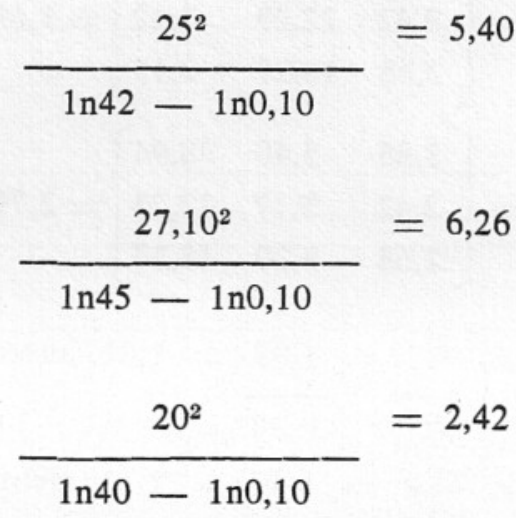

$x$
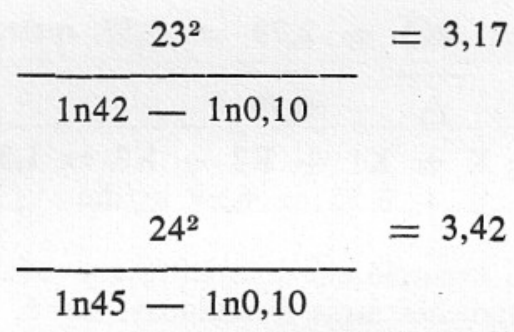

$x$

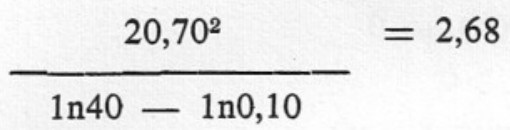

$\times \quad \frac{22,70^{2}}{\ln 42-1 \mathrm{n} 0,10}=3,20$

$x$

$$
=2,15
$$




\section{SISTEMA LINEAR DE EQUAÇÕES}

$3,85 \mathrm{~K}_{1}+5,40 \mathrm{~K}_{2}+6,26 \mathrm{~K}_{3}=38,94$

$2,42 \mathrm{~K}_{1}+3,17 \mathrm{~K}_{2}+3,42 \mathrm{~K}_{3}=22,23$

$2,68 \mathrm{~K}_{1}+3,20 \mathrm{~K}_{2}+2,15 \mathrm{~K}_{3}=18,37$

$$
\begin{aligned}
\Delta & =\left[\begin{array}{lll}
3,85 & 5,40 & 6,26 \\
2,42 & 3,17 & 3,42 \\
2,68 & 3,20 & 2,15
\end{array}\right]=0,79>0 \\
\Delta \mathrm{K}_{1} & =\left[\begin{array}{lll}
38,94 & 5,40 & 6,26 \\
22,23 & 3,17 & 3,42 \\
18,37 & 3,20 & 2,15
\end{array}\right]=1,09>0 \\
\Delta \mathrm{K}_{2} & =\left[\begin{array}{lll}
3,85 & 38,94 & 6,26 \\
2,42 & 22,23 & 3,42 \\
2,68 & 18,37 & 2,15
\end{array}\right]=1,69>0 \\
\Delta \mathrm{K}_{3} & =\left[\begin{array}{lll}
3,85 & 5,40 & 38,94 \\
2,42 & 3,17 & 22,23 \\
2,68 & 3,20 & 18,37
\end{array}\right]=2,79>0 \\
\mathrm{~K}_{1} & =\frac{\Delta \mathrm{K} 1}{\Delta}=\frac{1,09}{0,79}=1,32 \mathrm{metro} / \mathrm{dia} \\
\mathrm{K}_{2} & =\frac{\Delta \mathrm{K} 2}{\Delta}=\frac{1,69}{0,79}=2,14 \mathrm{metro} / \mathrm{dia} \\
\mathrm{K}_{3} & =\frac{\Delta \mathrm{K} 3}{2,79}=3,53 \mathrm{metro} / \mathrm{dia} \\
0,79 & \frac{\Delta}{\mathrm{K} 1}
\end{aligned}
$$

então $\mathrm{K}=\mathrm{K} 1+\mathrm{K} 2+\mathrm{K} 3=1,32+$ $+2,14+3,53=6,99 \mathrm{~m} / \mathrm{dia}$

No exemplo em questão foram satisfeitas as condições atrás formuladas, isto é:

$$
\begin{aligned}
& \mathrm{Q}_{1} \neq \mathrm{Q}_{2} \neq \ldots \ldots \ldots \mathrm{Q}_{\mathrm{n}} \\
& \mathrm{d}(\mathrm{A}) \neq 0 \\
& \mathrm{a}_{\mathrm{ik}}>0 \\
& \text { Sendo } \mathrm{K}_{1}>0, \\
& \text { então todos os produtos } \mathrm{a}_{\mathrm{ik}} \mathrm{K}_{\mathrm{i}}>0
\end{aligned}
$$

A possibilidade de aplicação do método exige certa uniformidade dos estratos de sedimentos e uma distância entre poços de no mínimo 100 metros, tendo em vista o raio de influência $R$.

Os sinais de $\Delta, \Delta_{K 1}, \Delta_{K 2}, \ldots \Delta_{K n}$ deverão ser idênticos para que não resul- tem $\mathrm{K}_{\mathrm{i}}<0$, pois neste caso não se cumpriria a relação $Q=\Delta Q_{i}$. Se algum $K_{1}<0$ for de valor pequeno, adota-se $K_{i}=0$, pois que o valor negativo resulta de inevitáveis imprecisões na avaliação das grandezas em jogo.

O método aqui proposto parte das seguintes suposições:

- a estrutura do aquífero tem certa uniformidade, isto é, as estratificações ocorrem segundo camadas dispostas mais ou menos horizontais e sedimentologicamente uniformes;

- cada camada de estratos se comporta como um aqǘf́ero independente de lençol livre;

- os componentes do aqǘf́rero obedecem a equação de Dupuit;

- o rebaixamento total se distribui proporcionalmente à espessura dos estratos sedimentares;

- o diâmetro do poço é constante e o raio de influência é tomado aproximadamente em função das frações de sedimentos de cada camada.

Estas hipóteses de base são evidentemente necessárias e são procedimentos correntes na formulação de qualquer lei que descreva fenômenos físicos.

Em conclusão o método deverá encerrar erros devidos às seguintes razões:

1. A descrição matemática do problema é inexata (por exemplo, os dados, iniciais não são exatos).

2. A solução exata requereria um número infinito e inaceitável de operações aritméticas, o que justifica a procura de soluções aproximadas, satisfatórias em face do problema considerado.

3. O inevitável erro de arredondamento de valores resultantes das operações.

Os erros devidos a estas causas são geralmente classificados como:

a) erros inerentes $r_{1}$,

b) erros de método $r_{2}$,

c) erros de cálculo $r_{3}$.

$O$ erro total resultante será então $r=$ $\mathrm{r}_{1}+\mathrm{r}_{2}+\mathrm{r}_{3}$.

Não obstante a lógica do método, existe a sua aplicabilidade, se viabiliza na medida das possibilidades da redução dos erros acima citados. 